6 CARDIAC MORBIDITY AND MORTALITY CAN BE ACCURATELY PREDICTED IN PATIENTS PRESENTING WITH ACS USING MULTIPLE BIOMARKERS MEASURED ON AN ADMISSION BLOOD SAMPLE

doi:10.1136/heartjnl-2011-300198.6

${ }^{1} \mathrm{R}$ Pearson, ${ }^{1} \mathrm{~K}$ Viswanathan, ${ }^{1} \mathrm{~N}$ Kilcullen, ${ }^{2} \mathrm{~A}$ S Hall, ${ }^{2} \mathrm{C}$ P Gale, ${ }^{1} \mathrm{U}$ M Sivananthan, ${ }^{1} \mathrm{~J} H$ Barth, ${ }^{2} \mathrm{C}$ Morrell. ${ }^{1}$ Leeds Teaching Hospitals, Leeds, UK; ${ }^{2}$ University of Leeds, Leeds, UK

Background Rapid assessment of patients with suspected acute coronary syndrome (ACS) allows the right patients to receive the right treatment at the right time. Discrimination of risk permits clinical triage into pathways of immediate inpatient or deferred outpatient care. It is known that a significant proportion of the ACS patients sent home following an "MI screen", based on a negative 12-h troponin level, are misdiagnosed as having noncardiac chest pain when in fact they are at high risk of cardiac events. It has been shown that the novel biomarker H-FABP can detect myocardial ischaemia even in the absence of myocyte necrosis. We hypothesise that a multi biomarker blood test incorporating troponin I, CK-MB and H-FABP, taken on admission, can accurately discriminate those patients with a non-cardiac cause of chest pain who are at low risk of cardiac morbidity or mortality.

Methods We studied 519 patients with suspected ACS admitted to a single UK Teaching Hospital. A risk scoring model was constructed based on tertile values for Randox Cardiac-Array measurement of troponin I, H-FABP and CK-MB. These were measured on a blood sample taken at the time of hospital admission. The lowest two lower tertiles were each given a score of 1 and the top tertile a score of 3 . The scores were then combined by summation resulting in an overall score of between 3 and 9 . Outcome measures up to 12 months were: (i) death from all causes; (ii) repeat acute coronary syndrome (ACS) (iii); readmission for heart failure; (iv) readmission for cerebrovascular event (CVA); (v) coronary revascularisation.

Results The distribution of Cardio-Array scores was: 3 ( $n=164$; $31.6 \%) ; 5$ ( $n=134 ; 25.8 \%) ; 7$ ( $n=110 ; 21.2 \%) ; 9$ ( $n=111 ; 21.4 \%)$. The cumulative incidence of events according to the Cardiac-Array score is shown in Abstract 6 table 1 .

Abstract 6 Table 1 The cumulative incidence of events according to the Cardiac-Array Score

\begin{tabular}{llllll}
\hline Score & Death & or ACS & or HF & or CVA & or Revasc \\
\hline 3 & $0.61 \%$ & $3.07 \%$ & $3.11 \%$ & $3.11 \%$ & $4.28 \%$ \\
5 & $3.21 \%$ & $5.77 \%$ & $5.81 \%$ & $5.81 \%$ & $6.41 \%$ \\
7 & $11.11 \%$ & $17.78 \%$ & $19.05 \%$ & $20.93 \%$ & $24.44 \%$ \\
9 & $12.98 \%$ & $16.23 \%$ & $18.37 \%$ & $18.92 \%$ & $22.08 \%$ \\
Ratio $(9 / 3)$ & 21.28 & 5.29 & 5.91 & 6.08 & 5.16 \\
p Value & $<0.0001$ & $<0.0001$ & $<0.0001$ & $<0.0001$ & $<0.0001$ \\
\hline
\end{tabular}

Conclusion Patients presenting with possible ACS who have a Cardiac-Array biomarker score of 3 or 5 , as measured on their admission blood sample, have a very low rate of cardiovascular events. This tool could be used to safely triage patients towards early discharge and outpatient care, based upon available resources. A score of 7 or 9 would merit admission to hospital, and consideration of early cardiac catheterisation.

\section{IN ACUTE CORONARY SYNDROMES, HEART-TYPE FATTY ACID BINDING PROTEIN IS A MORE ACCURATE PREDICTOR OF LONG TERM PROGNOSIS THAN TROPONIN}

doi:10.1136/heartjnl-2011-300198.7

${ }^{1}$ I R Pearson, ${ }^{2} \mathrm{~A}$ S Hall, ${ }^{2} \mathrm{C}$ P Gale, ${ }^{1} \mathrm{U}$ M Sivananthan, ${ }^{1} \mathrm{~K}$ Viswanathan, ${ }^{1} \mathrm{~N}$ Kilcullen, ${ }^{2} \mathrm{C}$ Morrell, ${ }^{1} \mathrm{~J} \mathrm{H}$ Barth. ${ }^{1}$ Leeds Teaching Hospitals, Leeds, UK; ${ }^{2}$ University of Leeds, Leeds, UK

Introduction We have previously shown that heart-type fatty acid binding protein (H-FABP) has a role in predicting all-cause mortality after acute coronary syndromes (ACS) and after multivariable analysis, provides additional information to that gained from the GRACE clinical risk factor score, troponin and highly sensitive CRP. H-FABP is released into the circulation during myocardial ischaemia and after myocardial necrosis, in contrast to troponin which is released after myocardial necrosis only. We have also shown that there is a group of ACS patients who are at high risk of cardiac events and death despite normal troponin levels on admission. This group may benefit from an early invasive strategy.

Hypothesis Plasma H-FABP level, taken between 12 and $24 \mathrm{~h}$ after admission, can identify troponin negative ACS patients who are at a high long term risk of death

Methods Six-year mortality data is now available for patients enrolled in the FAB 1 study, for which 1-year mortality data was published in 2007. In this study, 1448 unselected patients admitted to hospital with ACS had serum H-FABP level measured in addition to usual care. Mortality was tracked by the UK Office of National Statistics.

Results At 6 years overall all-cause mortality, available for 1421 patients $(98.1 \%)$, was $43.5 \%$. If troponin -ve/H-FABP -ve mortality was $20.9 \%$; troponin -ve/H-FABP +ve $56.4 \%$; troponin +ve/H-FABP -ve 20.2\%; troponin +ve/H-FABP +ve $49.1 \%$. Mortality rate was independent of troponin status but strongly related to $\mathrm{H}-\mathrm{FABP}$ status.

Conclusion The current system of stratification of ACS patients for early invasive management if troponin positive will miss a cohort of patients who are at high risk of death despite being troponin negative, and who may benefit from invasive investigation. Conversely, it is likely that some ACS patients undergo angiography based on a false positive troponin level. The addition of H-FABP measurement to the management of ACS could avoid this.

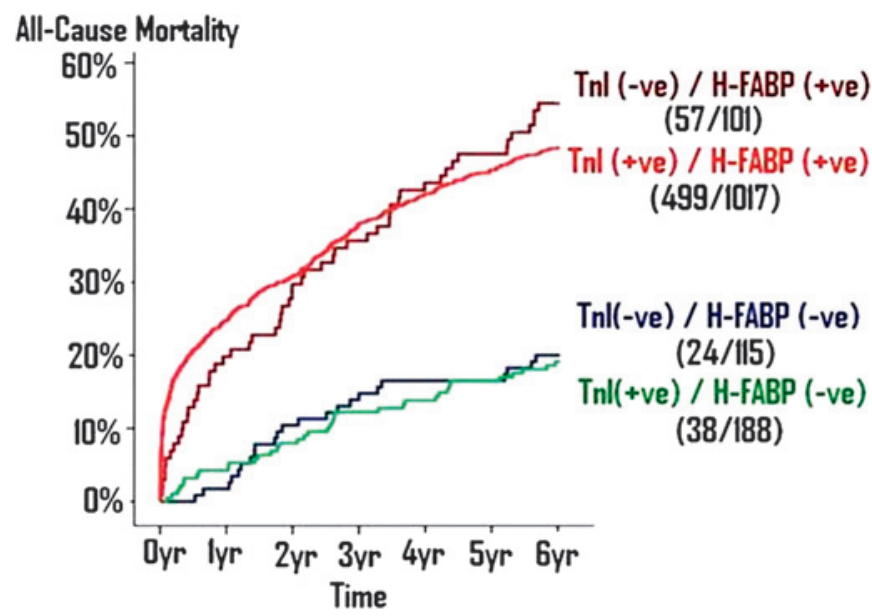

Abstract 7 Figure 1 